\title{
Acceptance and Commitment Therapy Delivered via a Mobile Phone Messaging Robot to Decrease Postoperative Opioid Use in Patients With Orthopedic Trauma: Randomized Controlled Trial
}

Chris A Anthony ${ }^{1,2}, \mathrm{MD}$; Edward Octavio Rojas ${ }^{3}, \mathrm{MD}$; Valerie Keffala ${ }^{3}, \mathrm{PhD}$; Natalie Ann Glass ${ }^{3}, \mathrm{PhD}$, MHCDS; Apurva S Shah ${ }^{4}$, MD, MBA; Benjamin J Miller ${ }^{3}$, MD, MS; Matthew Hogue ${ }^{3}$, MD; Michael C Willey ${ }^{3}$, MD; Matthew $\mathrm{Karam}^{3}$, MD; John Lawrence Marsh ${ }^{3}$, MD

\footnotetext{
${ }^{1}$ Department of Orthopaedics, University of Pennsylvania, Philadelphia, PA, United States

${ }^{2}$ Department of Orthopedic Surgery, Washington University School of Medicine, St Louis, MO, United States

${ }^{3}$ Department of Orthopaedics and Rehabilitation, University of Iowa Hospitals and Clinics, Iowa City, IA, United States

${ }^{4}$ Children's Hospital of Philadelphia Main Campus Division of Orthopaedics, Philadelphia, PA, United States
}

\section{Corresponding Author:}

Edward Octavio Rojas, MD

Department of Orthopaedics and Rehabilitation

University of Iowa Hospitals and Clinics

200 Hawkins Drive

Iowa City, IA, 52242

United States

Phone: 13193568690

Email: edward-rojas@uiowa.edu

\begin{abstract}
Background: Acceptance and commitment therapy (ACT) is a pragmatic approach to help individuals decrease avoidable pain. Objective: This study aims to evaluate the effects of ACT delivered via an automated mobile messaging robot on postoperative opioid use and patient-reported outcomes (PROs) in patients with orthopedic trauma who underwent operative intervention for their injuries.

Methods: Adult patients presenting to a level 1 trauma center who underwent operative fixation of a traumatic upper or lower extremity fracture and who used mobile phone text messaging were eligible for the study. Patients were randomized in a 1:1 ratio to either the intervention group, who received twice-daily mobile phone messages communicating an ACT-based intervention for the first 2 weeks after surgery, or the control group, who received no messages. Baseline PROs were completed. Two weeks after the operative intervention, follow-up was performed in the form of an opioid medication pill count and postoperative administration of PROs. The mean number of opioid tablets used by patients was calculated and compared between groups. The mean PRO scores were also compared between the groups.

Results: A total of 82 subjects were enrolled in the study. Of the 82 participants, 76 ( 38 ACT and 38 controls) completed the study. No differences between groups in demographic factors were identified. The intervention group used an average of 26.1 (SD 21.4) opioid tablets, whereas the control group used 41.1 (SD 22.0) tablets, resulting in 36.5\% ([41.1-26.1]/41.1) less tablets used by subjects receiving the mobile phone-based ACT intervention $(P=.004)$. The intervention group subjects reported a lower postoperative Patient-Reported Outcome Measure Information System Pain Intensity score (mean 45.9, SD 7.2) than control group subjects (mean 49.7, SD 8.8; $P=.04$ ).

Conclusions: In this study, the delivery of an ACT-based intervention via an automated mobile messaging robot in the acute postoperative period decreased opioid use in selected patients with orthopedic trauma. Participants receiving the ACT-based intervention also reported lower pain intensity after 2 weeks, although this may not represent a clinically important difference.
\end{abstract}

Trial Registration: ClinicalTrials.gov NCT03991546; https://clinicaltrials.gov/ct2/show/NCT03991546

(J Med Internet Res 2020;22(7):e17750) doi: 10.2196/17750 


\section{KEYWORDS}

acceptance and commitment therapy; opioid crisis; patient-reported outcome measures; postoperative pain; orthopedics; text messaging; chatbot; conversational agents; mHealth

\section{Introduction}

\section{Opioid Medication Issues}

Public health concerns regarding opioid medications persist, and health care systems are currently seeking solutions to the ongoing epidemic [1]. In 2017, the rate of drug overdose deaths involving opioids in the United States increased by $12 \%$, totaling 47,600 cases, and prescription opioid medications accounted for over 17,000 of these [2]. Even small amounts of additional daily opioid utilization (10 morphine milliequivalents [MME]) by patients can lead to an increased risk of long-term misuse [3]. In addition, every week of continued opioid utilization represents an increased risk of eventual misuse by patients [3]. Previous studies have found that orthopedic trauma patients use a decreasing amount of opioid medication in the first 2 postoperative weeks, with 6-15 days being the optimal opioid use period [4,5]. In line with these findings, previous studies have used a 2-week postoperative period to assess opioid medication consumption in surgical patients [4].

\section{Patient-Reported Outcomes}

Patient-reported outcomes (PROs) allow patients to quantify aspects of their orthopedic condition in a standardized fashion $[6,7]$. These are important tools for determining the efficacy of health care treatments and assessment of clinical research and can be used in determining compensation for health care services provided [6-8]. The National Institutes of Health developed the Patient-Reported Outcome Measure Information System (PROMIS) tools to advance PROs by creating question banks that could be used for many major health issues [9]. The
PROMIS Pain Intensity 1A Short form, PROMIS Pain Intensity 3A Short form, PROMIS Pain Interference 8A Short form, and PROMIS Emotional Distress-Anxiety 8A Short form all employ a fixed low number of questions that are highly reliable when compared with their respective domain's full item bank, making them excellent tools for both patients and clinicians $[9,10]$.

\section{Acceptance and Commitment Therapy}

Acceptance and commitment therapy (ACT) is a cognitive contextual behavioral therapy that employs a pragmatic approach to help individuals decrease pain and live according to self-identified personal values $[11,12]$. The goal of ACT is to augment an individual's psychological flexibility, thus improving their life according to 6 core cognitive processes: acceptance, defusion, contact with the present moment, self-as-context, values, and committed action (Table 1) [11]. Employing these cognitive processes to increase psychological flexibility allows people to choose their actions based on what they value most, resulting in decreased avoidance behaviors and negative cognitive associations [11]. ACT has proven to be effective across multiple studies and patient populations in the treatment of pain [13]. Several studies report a high value for ACT in the management of chronic pain when compared with standard pharmacological treatment alone [13-16]. Moreover, earlier cessation of pain and opioid utilization in at-risk orthopedic surgery patients receiving office-based ACT interventions has also been reported [17]. However, traditional ACT interventions require a clinic-based, interdisciplinary team approach, which is not always feasible for both patients and health care systems $[17,18]$. 
Table 1. Acceptance and commitment therapy core principles with associated messages.

\begin{tabular}{|c|c|}
\hline Core principle & Example mobile phone message \\
\hline $\begin{array}{l}\text { Values: know what matters } \\
\text { most }\end{array}$ & $\begin{array}{l}\text { Stop for a moment and remember the } 3 \text { values you identified earlier today. Remind yourself how important these values } \\
\text { are in your life. As your day comes to an end, remember that YOU are in control of the thoughts that exist in your mind. } \\
\text { We encourage you to spend time thinking about your } 3 \text { core values identified earlier today. }\end{array}$ \\
\hline $\begin{array}{l}\text { Acceptance: setting expecta- } \\
\text { tion that pain is a part of } \\
\text { surgery }\end{array}$ & $\begin{array}{l}\text { Feelings of pain and feelings about your experience of pain are normal after surgery. Acknowledge and accept these } \\
\text { feelings as part of the recovery process. Remember how you feel now is temporary and your healing process will con- } \\
\text { tinue. Call to mind pleasant feelings or thoughts that you experienced today. }\end{array}$ \\
\hline $\begin{array}{l}\text { Present moment awareness: } \\
\text { mindfulness and awareness } \\
\text { for our thoughts in the } \\
\text { present moment }\end{array}$ & $\begin{array}{l}\text { Awareness of the present moment and your breathing may change with pain-related emotions or thoughts. Remember } \\
\text { you can always count on your breathing to bring you back to the present moment and help you move through your } \\
\text { current experience of pain. }\end{array}$ \\
\hline $\begin{array}{l}\text { Self-as-context: awareness } \\
\text { of what is being observed } \\
\text { and noticed by ourselves }\end{array}$ & $\begin{array}{l}\text { We cannot change that a feeling or thought may arise, but we can choose how we respond to our feelings and thoughts. } \\
\text { Remember that dwelling on pain, discouraging feelings, and thoughts after surgery are NOT consistent with your life } \\
\text { goals and values. Observe things that try to move you away from your values and only act on things that are compatible } \\
\text { with who you want to be and what matters to you. }\end{array}$ \\
\hline $\begin{array}{l}\text { Committed action: doing } \\
\text { what it takes to live accord- } \\
\text { ing to our values }\end{array}$ & $\begin{array}{l}\text { Healing after surgery requires you to act. We previously discussed your life goals, meaning, and purpose. Take action } \\
\text { today and move closer toward what you want in life. Recognize that pain may be present but make the choice that it } \\
\text { will not impede your progress toward what you really want in life. Be present in the moment and ensure your actions } \\
\text { remain true to what you want most. All actions you make no matter how small, are an important steppingstone on your } \\
\text { road to recovery. }\end{array}$ \\
\hline $\begin{array}{l}\text { Defusion: watch your think- } \\
\text { ing and interact with } \\
\text { thoughts in a way consistent } \\
\text { with your values }\end{array}$ & $\begin{array}{l}\text { If you ever feel pain after surgery know that the feeling is real but what it actually represents is not what you might } \\
\text { think. Our mind is capable of making us feel pain, even though there is no damage going on in our body. Pause, become } \\
\text { more aware in the moment and chose a skillful response that will help you move toward your overall goals and values. }\end{array}$ \\
\hline
\end{tabular}

\section{Mobile Phone Messaging Communication}

Evolving communication methods, such as automated mobile phone messaging [4,19-21], for health care purposes are increasingly important, as patients prefer these communication methods for delivering and receiving medical information [22]. Software-driven, automated mobile phone messaging robots (also called chatbots or conversational agents) are low-cost tools that can deliver predefined text-based information and receive incoming responses with high reliability when patients either prefer it or it is necessary to communicate at distance $[19,23]$. This technology demonstrates high efficacy as part of the treatment of conditions ranging from hypertension to substance abuse [24-26], and it has also proved effective in increasing perioperative communication after hip and knee arthroplasty [27] and collecting pain and opioid medication data from patients following orthopedic trauma and hand procedures $[4,19]$. In addition, automated mobile phone messaging robots have been validated in the collection of PROs in patients undergoing orthopedic hand [20] and hip preservation procedures [23]. Although mobile phone messaging robots (Chat bots) provide the benefit of communicating with patients at distance with no need for human intervention, they do introduce delivery of health care that lacks human interaction with unknown effects [28].

Health care teams caring for patients with traumatic orthopedic injuries have traditionally used opioid medication in the postoperative setting, and these patients are at risk for prolonged opioid utilization in the postoperative period. We theorized that the combination of ACT delivered via automated mobile phone messaging may help to decrease pain and opioid utilization in the acute postoperative setting. The aim of this prospective randomized controlled trial was to evaluate the effectiveness of
ACT delivered via an automated mobile messaging robot on (1) decreasing early postoperative opioid utilization and (2) pain-related PROs in the first 2 weeks following surgery for acute traumatic orthopedic injuries.

\section{Methods}

\section{Study Approval}

This randomized controlled trial was registered with ClinicalTrials.gov (NCT03991546) and reporting is consistent with the Consolidated Standards of Reporting Trials guidelines (Multimedia Appendix 1) [29]. The study was performed at a single center university hospital in Iowa City, Iowa, United States. Ethical approval of this study was provided by the University of Iowa institutional review board, and the study was determined to be Health Insurance Portability and Accountability Act compliant.

\section{Recruitment and Randomization}

Adults presenting to a university hospital level 1 trauma center indicated for operative fixation of a traumatic upper or lower fracture were considered for the study (Table 2). The exclusion criteria are listed in Textbox 1. Eligible patients were approached before surgery by a research assistant in a private room. Individuals not excluded by screening questions and interested in participating underwent the informed consent process (Textbox 1). During consent, all subjects were informed of the outcomes of interests, different study arms, and that no changes would be made to their care in terms of postoperative medication, regardless of study participation.

Participants were randomized to either the control or intervention group using a standard web-based random number generator with a range set from 1 to 10 and a 1:1 ratio by a 
research assistant. Owing to the nature of this study, subjects and the enrolling research assistant were not blinded to the participant's study group following randomization.

At the time of consent, subjects were required to complete paper forms comprising a basic demographics questionnaire and baseline PROs consisting of the PROMIS Pain Intensity 1A Short form, PROMIS Pain Intensity 3A Short form, PROMIS
Pain Interference 8A Short form, and PROMIS Emotional Distress-Anxiety 8A Short form (Multimedia Appendix 2). Following completion of all PROs, participants were randomized to their study group. Subjects who received an odd number from the 1 to 10 range set on the random number generator were placed in the intervention group, whereas subjects given an even number were placed in the control group.

Table 2. Injury by final study group $(\mathrm{N}=76)$.

\begin{tabular}{lll}
\hline Injury & Acceptance and commitment therapy group participants, $\mathrm{n}$ & Control group participants, $\mathrm{n}$ \\
\hline Acetabular fracture & 1 & 1 \\
Ankle fracture & 15 & 14 \\
Calcaneus fracture & 0 & 1 \\
Clavicle fracture & 0 & 1 \\
Distal femur fracture & 0 & 2 \\
Distal humerus fracture & 1 & 0 \\
Elbow fracture & 2 & 5 \\
Femoral neck fracture & 2 & 2 \\
Femoral shaft stress fracture & 0 & 1 \\
Intertrochanteric hip fracture & 1 & 0 \\
Navicular fracture & 1 & 0 \\
Patella fracture & 1 & 0 \\
Polytrauma & 2 & 1 \\
Proximal humerus fracture & 2 & 1 \\
Subtrochanteric femur fracture & 0 & 2 \\
Tibial plateau fracture & 4 & 3 \\
Tibial plafond fracture & 6 & 4 \\
\hline
\end{tabular}

${ }^{\text {a }}$ Polytrauma was defined as a patient with a fracture to more than one upper or lower extremity.

Textbox 1. Exclusion criteria.

Screening questions

- No personal mobile phone with text messaging capabilities

- Poor familiarity reading or sending mobile messages

Patient factors

- Open fracture

- Infection at the fracture site

- Prior fracture temporization with an external fixator

- Revision surgery for nonunion or hardware failure

- Bilateral upper extremity injuries impeding their ability to use a mobile phone

- Fractures of the distal hand or distal foot only

- Admission to an intensive care unit

- Current cancer diagnosis or dementia

- Inpatient for more than 7 days of the 2-week study period

- Discharged without an opioid pain medication prescription

- Initial plan for operative fixation changed to treatment with joint arthroplasty 


\section{Study Interventions}

The intervention group received twice-daily, text-based mobile messages communicating an ACT-based intervention for the first 2 weeks following surgery (Multimedia Appendix 3). Control group subjects did not receive the ACT intervention or any other form of mobile message communication. The mobile messaging ACT protocol consisted of twice per day mobile messages, morning and evening, starting on postoperative day (POD) 1 and ending on POD 14. These mobile phone messages provided participants with an ACT-based intervention that was developed in collaboration with a pain psychologist (VK) specializing in ACT for chronic pain. These messages used all the principles presented in Table 1 with the objective of helping recipients understand and develop better coping skills in relation to their postoperative pain. An example message from day 1 is as follows:

Maintaining focus on what you value most in life is sometimes difficult after surgery. Do not let the momentary discomforts due to surgery take away from what you want most in life. Pick 3 things that matter most to you in life. Remind yourself of these 3 things you value most during your recovery process.

Outside of the mobile messaging intervention, both groups received the same standard postoperative care, health care team communications, and instructions for completing the study follow-up.

A chart review was performed to collect demographic information such as subject age, comorbid conditions, and preoperative outpatient opioid medication prescriptions for treatment of their current traumatic orthopedic injury. All subjects, regardless of group, were seen by a research team member after surgery to review which of their discharge medications was the medication of interest for the study and to confirm that the intervention group subjects received their first mobile phone ACT message. Participants in both groups were instructed to have their opioid medication bottle available at follow-up to confirm their opioid tablet consumption. Owing to the changes in health care teams, staff preferences, and allergies, the opioid pain medications administered at discharge were not standardized between study groups. Following discharge on POD 14, subjects were contacted by phone or seen in the clinic by the research team for follow-up. At this time, the subjects' opioid pain medication consumption was assessed, and they completed a second set of PROs.

\section{Outcome Measures}

The primary outcome of this study was the amount of opioid pain medication consumed by subjects, and the secondary outcomes analyzed were net changes from baseline PRO scores at the 2-week follow-up.

The method that participants employed to report their opioid medication consumption and how PROs were captured during follow-up were recorded (Table 3). Subjects using their pill bottle to confirm the remaining number of opioid pain medication tablets from their discharge prescription on POD 14 were denoted as reporting a pill count. Cases where subjects or their care facility kept a log of tablet consumption were classified as reporting a daily log. Subjects reporting the number of tablets they used without the use of a log or pill count were designated as providing an estimate. The percentage of opioid pain medication used, total MME, and percentage of available MME consumed were calculated. The mean number of opioid pain medication tablets and MME used by the subjects were compared between groups. The raw scores for PROs were converted to corresponding $t$-scores using the appropriate PROMIS scoring manual [30]. The changes in PROs from baseline to POD 14 were also calculated by subtracting POD 14 scores from baseline scores, as higher $t$-scores signify a poorer outcome; thus, lower scores on POD 14 indicate an improvement from baseline PROs. The mean PRO scores and changes were compared between the groups. 
Table 3. Comparison of subject demographics by enrolled study group.

\begin{tabular}{|c|c|c|c|}
\hline Subject characteristic & Acceptance and commitment therapy group $(n=42)$ & Control group $(n=40)$ & $P$ value \\
\hline Age (years), mean (SD) & $45.5(15.9)$ & $48.7(14.6)$ & .41 \\
\hline BMI $\left(\mathrm{kg} / \mathrm{m}^{2}\right)$, mean $(\mathrm{SD})$ & $30.5(7.3)$ & $31.1(8.3)$ & .94 \\
\hline $\operatorname{Sex}, \mathbf{n}(\%)$ & & & .65 \\
\hline Female & $22(52)$ & $19(48)$ & \\
\hline Male & $20(48)$ & $21(52)$ & \\
\hline Subjects removed or lost to follow-up, n (\%) & $4(10)$ & $2(5)$ & $\mathrm{N} / \mathrm{A}^{\mathrm{a}}$ \\
\hline $\begin{array}{l}\text { Preoperative PROMIS }{ }^{\mathrm{b}} \text { Pain Intensity } 1 \mathrm{~A} \text { Score, } \\
\text { mean (SD) }\end{array}$ & $5.4(2.9)$ & $6.2(2.6)$ & .20 \\
\hline $\begin{array}{l}\text { Preoperative PROMIS Pain Intensity 3A Score, mean } \\
\text { (SD) }\end{array}$ & $54.9(7.3)$ & $57.1(8.2)$ & .23 \\
\hline $\begin{array}{l}\text { Mean Preoperative PROMIS Pain Interference 8A } \\
\text { Score, mean (SD) }\end{array}$ & $63.6(11.4)$ & $66.1(8.4)$ & .30 \\
\hline $\begin{array}{l}\text { Mean Preoperative PROMIS Emotional Distress- } \\
\text { Anxiety 8A Score, mean (SD) }\end{array}$ & $56.5(11.4)$ & $56.5(9.2)$ & .99 \\
\hline Days between injury and surgery, mean (range) & $4(1-33)$ & $3(1-50)$ & .26 \\
\hline Disposition $^{\mathrm{c}}, \mathbf{n}(\%)$ & & & .68 \\
\hline Home & $36(95)$ & $34(90)$ & \\
\hline Skilled nursing facility or acute rehabilitation & $2(5)$ & $4(10)$ & \\
\hline Ethnicity/race, n (\%) & & & .86 \\
\hline White & $37(88)$ & $35(88)$ & \\
\hline African American & $4(10)$ & $4(10)$ & \\
\hline Asian & $1(2)$ & $0(0)$ & \\
\hline Hispanic & $0(0)$ & $1(2)$ & \\
\hline Preoperative outpatient opioid prescription, n (\%) & $23(55)$ & $17(43)$ & .17 \\
\hline Current psychiatric diagnosis, n (\%) & $15(36)$ & $9(23)$ & .14 \\
\hline History/current substance abuse diagnosis, n (\%) & $8(19)$ & $3(8)$ & .10 \\
\hline Diabetes diagnosis, $\mathrm{n}(\%)$ & $2(5)$ & $7(18)$ & .15 \\
\hline Current smoker, n (\%) & $7(17)$ & $9(23)$ & .57 \\
\hline Current lumbago diagnosis, $\mathrm{n}(\%)$ & $1(2)$ & $2(5)$ & $>.99$ \\
\hline History of/current chronic pain diagnosis, $\mathrm{n}(\%)$ & $10(24)$ & $8(20)$ & .59 \\
\hline Number of opioid tablets prescribed ${ }^{\mathrm{c}}$, mean (SD) & $58.8(27.3)$ & $61.6(22.0)$ & .62 \\
\hline Opioid utilization reporting $\operatorname{method}^{\mathrm{c}}, \mathbf{n}(\%)$ & & & .47 \\
\hline Pill count & $34(90)$ & $30(79)$ & \\
\hline Daily log & $3(8)$ & $6(16)$ & \\
\hline Estimate & $1(2)$ & $2(5)$ & \\
\hline $\begin{array}{l}\text { Patients filling only one postoperative opioid prescrip- } \\
\text { tion }^{\mathrm{c}}, \mathrm{n}(\%)\end{array}$ & $34(90)$ & $34(90)$ & $>.99$ \\
\hline
\end{tabular}

${ }^{\mathrm{a}} \mathrm{N} / \mathrm{A}$ : not applicable.

${ }^{b}$ PROMIS: Patient-Reported Outcome Measures Information System.

${ }^{\mathrm{c}}$ Data calculated using final study population only $(\mathrm{n}=38)$. 


\section{Statistical Analysis}

Participant characteristics were described using mean (SD) or median (minimum to maximum) for continuous variables and frequencies (percentages) for categorical variables. Visual review of histograms and the results of the Shapiro-Wilk test of continuous variables revealed that only age and BMI were not normally distributed. Between-group differences were evaluated using $t$ tests or Wilcoxon rank-sum tests (age and BMI) for continuous variables and chi-square or exact tests for categorical variables, as appropriate.

To evaluate whether the intervention versus control group had a lower opioid use on average, we determined the number of tablets and MME taken in each group and compared means using $t$ tests. Using a previous study of opioid medication usage in orthopedic trauma patients [4], the sample size estimated to observe a $30 \%$ decrease in opioid utilization among 2 groups required a total of 74 subjects to achieve $80 \%$ power at an alpha of .05 . The percent decrease is calculated using the formula $\left(\frac{A-B}{A}\right) \times 100=$ percent decrease . A separate power analysis was calculated for the PRO portion of the study, and it was determined that a total of 36 subjects would provide $80 \%$ power to detect a 10 -point difference (1 standard deviation) in $t$-scores for the PROMIS instruments at an alpha level of .05. Statistical analyses were performed using SAS software version 9.4 (SAS Institute, Inc).

\section{Results}

\section{Study Participants}

A total of 125 individuals were approached regarding the study over the 5-month enrollment period between February 2019 and June 2019. Of the 125 individuals, 2 patients were excluded at this time, as they were non-English-speaking, and an additional 24 patients were excluded because they did not use mobile phone messaging or did not have a personal mobile phone. This resulted in a total of 99 eligible people who were presented the study, 17 of whom declined participation (Figure 1). Overall, 82 subjects were enrolled, and 6 dropped from the study after providing consent because of various issues: one patient lost to follow-up, one patient withdrew at follow-up, one patient had incomplete follow-up, one patient's operative plan changed to arthroplasty, and 2 subjects remained inpatient for over 7 days of the study period (Figure 1). This resulted in a final population of 76 subjects ( 38 per study group). The enrollment period concluded once a powered sample for the primary aim was obtained. A breakdown of the subjects enrolled, reasons for excluding subjects, and subjects removed from the study after consent are presented in Figure 1. Participant demographics for the intervention and control groups are presented in Table 3 . The analyses of all collected demographic factors showed no differences between the intervention and control groups in all factors such as subject age $(P=.42)$, current psychiatric diagnosis (including depression, anxiety, bipolar type 1, obsessive-compulsive disorder, posttraumatic stress disorder, panic disorder, and attention-deficit disorder; $P=.14$ ), or substance abuse history $(P=.10$; Table 3$)$. Furthermore, no differences between groups were found for injury type, disposition following discharge, method for reporting opioid medication consumption, preoperative opioid medication prescriptions, or preoperative PROs (Table 3). 
Figure 1. Consolidated Standards of Reporting Trials flowchart detailing the selection of eligible patients for study enrollment and their status through study completion. ACT: acceptance and commitment therapy.

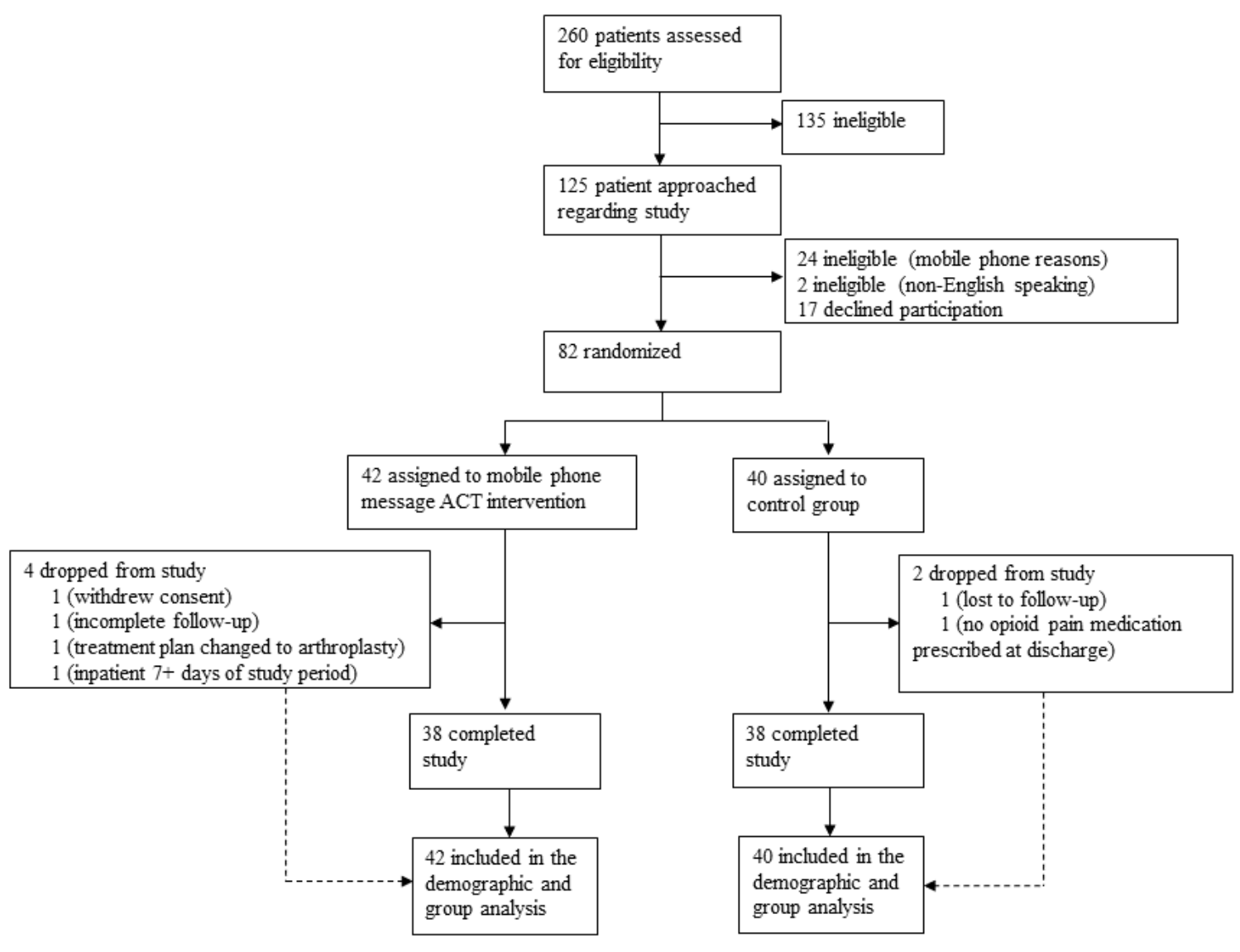

\section{Opioid Pain Medication Use}

No differences between groups were observed in the amount of opioid medication tablets or MME prescribed at discharge (tablets for the ACT group: mean 58.8, SD 27.3 vs tablets for the control group: mean 61.6, SD 22.0). A further breakdown of the medications prescribed to subjects within the study period is presented in Table 4 . The mean opioid tablet use for subjects in the ACT-based intervention group was 26.1 (SD 21.4) tablets, whereas the control group used a mean of 41.1 (SD 22.0) tablets, resulting in $36.5 \%$ less tablets used by subjects receiving the ACT-based intervention ( $P=.004$; Table 5). Similarly, subjects in the intervention group consumed a mean of 199.9 (SD 163.2) MME, on average, whereas the control group subjects consumed a mean of 307.0 (SD 166.0) MME, indicating 34.9\% less MME used by subjects in the intervention group ( $P=.006$; Table 5).

Table 4. Frequency of outpatient opioid pain medications prescribed by enrolled study group ( $\mathrm{N}=82)$.

\begin{tabular}{llll}
\hline Medication & Morphine milliequivalents per tablet & Frequency \\
& & Acceptance and commitment therapy & Control \\
\hline Hydrocodone-acetaminophen 5-325 mg & 5 & 1 & 2 \\
Hydrocodone-acetaminophen 10-325 mg & 10 & 2 & 0 \\
Hydromorphone 2 mg & 8 & 6 & 6 \\
Oxycodone 5 mg & 7.5 & 27 & 27 \\
Oxycodone-acetaminophen 5-325 mg & 7.5 & 46 & 43 \\
Total opioid prescriptions provided & $\mathrm{N} / \mathrm{A}^{\mathrm{a}}$ & & 40 \\
\hline
\end{tabular}

${ }^{\mathrm{a}} \mathrm{N} / \mathrm{A}$ : not applicable. 
Table 5. Opioid pain medication utilization by group during the 2-week study period.

\begin{tabular}{|c|c|c|c|c|c|c|c|c|c|c|c|}
\hline \multirow[t]{2}{*}{ Attribute } & \multicolumn{3}{|c|}{ Opioid tablets dispensed } & \multicolumn{4}{|c|}{ Opioid tablets consumed } & \multicolumn{4}{|c|}{ Morphine milliequivalents consumed } \\
\hline & $\begin{array}{l}\mathrm{ACT}^{\mathrm{a}} \\
(\mathrm{n}=38)\end{array}$ & $\begin{array}{l}\text { Control } \\
(n=38)\end{array}$ & $P$ value & $\begin{array}{l}\text { ACT } \\
(n=38)\end{array}$ & $\begin{array}{l}\text { Control } \\
(\mathrm{n}=38)\end{array}$ & $\begin{array}{l}\text { Decrease }^{\mathrm{b}} \\
(\%)\end{array}$ & $P$ value & $\begin{array}{l}\text { ACT } \\
(n=38)\end{array}$ & $\begin{array}{l}\text { Control } \\
(\mathrm{n}=38)\end{array}$ & $\begin{array}{l}\text { Decrease } \\
(\%)\end{array}$ & $P$ value \\
\hline Mean (SD) & $\begin{array}{l}58.8 \\
(27.3)\end{array}$ & $\begin{array}{l}61.6 \\
(22.0)\end{array}$ & .62 & $\begin{array}{l}26.2 \\
(21.4)\end{array}$ & $\begin{array}{l}41.1 \\
(22.0)\end{array}$ & 37 & .004 & $\begin{array}{l}199.9 \\
(163.2)\end{array}$ & $\begin{array}{l}307.0 \\
(166.0)\end{array}$ & 35 & .006 \\
\hline $\begin{array}{l}\text { Median } \\
\text { (minimum- } \\
\text { maximum) }\end{array}$ & $\begin{array}{l}60.0 \\
(10- \\
146)\end{array}$ & $\begin{array}{l}60.0 \\
(15- \\
120)\end{array}$ & .62 & $\begin{array}{l}21.0(0- \\
80)\end{array}$ & $\begin{array}{l}43.5(0- \\
80)\end{array}$ & 37 & .004 & $\begin{array}{l}157.5 \\
(0-600)\end{array}$ & $\begin{array}{l}307.5 \\
(0-600)\end{array}$ & 35 & .006 \\
\hline
\end{tabular}

aCT: acceptance and commitment therapy.

${ }^{\mathrm{b}}$ Calculated by the formula $\left(\frac{A-B}{A}\right) \times 100=$ percent decrease .

\section{Patient-Reported Outcomes}

PROMIS instrument $t$-score values for both ACT and control group subjects are presented in Table 6. At 2-week follow-up, the intervention group subjects reported lower postoperative PROMIS Pain Intensity 3A (mean 45.9, SD 7.2) and Pain
Interference 8A (mean 56.6, SD 9.4) scores compared with the control group's postoperative Pain Intensity 3A (mean 49.7, SD $8.8 ; P=.04$ ) and Pain Interference 8 A scores (mean 60.6, SD $8.2 ; P=.05$; Table 6 ). No differences were observed between groups at 2-week follow-up in the PROMIS Pain Intensity $1 \mathrm{~A}$ or PROMIS Emotional Distress-Anxiety 8A forms (Table 6).

Table 6. Mean Patient-Reported Outcome Measures Information System score and change within the 2-week study period by study group.

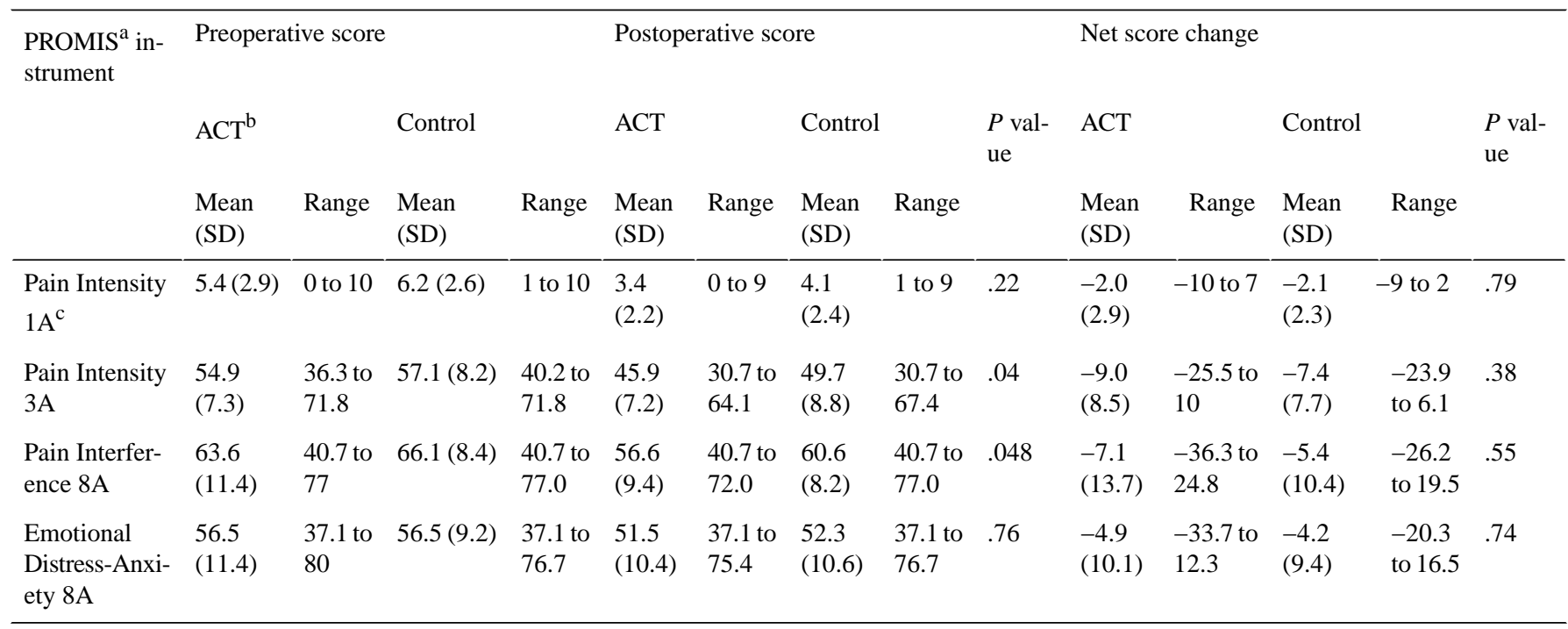

${ }^{\text {a} P R O M I S: ~ P a t i e n t-R e p o r t e d ~ O u t c o m e ~ M e a s u r e s ~ I n f o r m a t i o n ~ S y s t e m . ~}$

${ }^{\mathrm{b}} \mathrm{ACT}$ : acceptance and commitment therapy.

${ }^{\mathrm{c}}$ Scores presented are raw numerical scores, as no $t$-score conversion is available for the selected instrument.

\section{Discussion}

\section{Principal Findings}

This randomized trial delivered an ACT-based intervention via an automated mobile messaging robot to postoperative orthopedic patients. The subjects who received the ACT-based mobile phone intervention used a lower number of opioid tablets and consumed less MME in the first 2 weeks after their injury. We also found that the intervention group reported less pain intensity and pain interference at the 2-week follow-up. These data demonstrate that ACT-based automated mobile messaging protocols may be effective in reducing the amount of opioid medication used and may positively affect postoperative PROs in patients undergoing operative fixation of their acute fractures.

\section{Effects on Opioid Use}

Improved mood symptoms, less pain interference, and faster cessation of opioid pain medication are some of the recognized benefits of using ACT in clinic-based, interdisciplinary approaches to pain management after surgery $[17,18]$. Previous investigations have used automated mobile phone messaging robots to deliver PROs [20,23], improve communication with patients [27,31], deliver postoperative orthopedic care [32], and inquire about pain and opioid utilization $[4,19,33]$. In this study, we used ACT and a mobile phone messaging robot to assess whether these tools in combination could decrease opioid utilization and improve individuals' perception of their early recovery from injury. Prior work has demonstrated a quicker time to opioid cessation and a decrease in postoperative opioid utilization (14\% less in the ACT group) when used in office ACT-based treatments $[17,18]$. Subjects receiving the ACT 
intervention via automated mobile phone messages reported over $36 \%$ less opioid tablets and more than $34 \%$ less MME consumed than corresponding control subjects who did not receive ACT. Our findings suggest that software-based communication using ACT through a mobile phone has the potential to have a large impact on the utilization of postoperative pain medication by patients in the first weeks after surgery for fractures. Further study is required to determine if these effects are long lasting and to determine which injuries and patients receive the greatest benefit. In addition, future investigations and trials should consider the effect of software delivery of ACT and other behavioral therapies on different cohorts of patients.

\section{Effects on PROs}

PROs, such as PROMIS, allow patients to quantify aspects of their orthopedic condition in a standardized fashion [6,7]. These are important tools for determining the efficacy of health care treatments and assessment of clinical research and can be used in determining compensation for health care services provided [6-8]. The National Institutes of Health developed the PROMIS tools to advance PROs by creating question banks that could be used for many major health issues [9]. We found that despite less utilization of opioids, subjects in the ACT-based intervention group reported less pain intensity and pain interference at 2 weeks. This most likely does not represent a clinically important difference based on the SD methodology used in prior works with PROMIS tools (Table 6), but it at least suggests that the intervention group did not experience greater pain [34]. There were no other differences between study groups in the other domains at the 2-week follow-up. Previous studies have reported that patients who consume more opioid medication report higher pain at both short- and long-term time points, which is reflected in our findings for both PROMIS pain intensity $3 \mathrm{~A}$ and the employed pain interference measure $[35,36]$. Future research efforts may benefit from employing alternative PRO measures to identify the effects of ACT-based interventions, including assessment of psychologic flexibility. Future research may also consider possible modifications of our study protocol to include a longer intervention period and more than one follow-up data point. Future work may also consider designing an ACT-based tool that is more focused on demonstrating an effect on PROs.

\section{Limitations}

Several limitations were present in this study. First, we were limited to a single level 1 trauma center, which may affect the reproducibility of our results across other health care settings. Next, the exclusion criteria for this study were extensive, and thus, the results may not be generalizable to the entire scope of orthopedic trauma patients. We attempted to include a diverse set of injuries and yet excluded patients with a high likelihood of confounding problems from open fractures or prolonged initial hospitalization. Future studies assessing the effects of ACT-based interventions similar to ours should aim for less restrictive exclusion criteria to apply this intervention to a larger, more diverse population. The research assistants were not blinded to the patients' study group. In addition, patients understood the outcomes of interest in this study, which could be susceptible to reporting bias. In addition, participants were not blinded to their treatment group. The lack of blinding could potentially introduce response or reporting bias, making this a potential area of improvement for studies seeking to follow the present methodology. This could be accomplished through the implementation of a control messaging protocol. Moreover, a retrospective chart review was used to obtain several patient factors, including comorbid conditions and dispensing of preoperative outpatient opioid medication prescriptions. The collection of information in this manner relies on accurate charting and transfer of documents from outside institutions, which may have been incomplete.

\section{Conclusions}

In this study, delivering an ACT-based intervention via an automated mobile messaging robot in the acute postoperative period decreased opioid utilization in orthopedic trauma patients in the first 2 weeks after their injury. Subjects in the ACT-based intervention group also reported lower pain intensity and pain interference after 2 weeks, although this likely did not represent a clinically important difference. Future studies may apply this intervention in other patient populations to assess its efficacy on a larger scale and may include assessment of pain and opioid use in a longer time frame after injury.

\section{Acknowledgments}

This study was possible thanks to a generous grant from the Orthopaedic Trauma Association.

\section{Conflicts of Interest}

CA reports personal fees from McKinsey \& Company, outside the submitted work. MW reports nonfinancial support from Zimmer Biomet, outside the submitted work. MK reports stock or stock options from Iowa Simulation Solutions LLC and stock or stock options from Mortise Medical LLC, outside the submitted work. JM reports stock or stock options from Zimmer Biomet, stock or stock options from FxRedux, nonfinancial support and stock or stock options from Oxford Press, and stock or stock options from Tornier, outside the submitted work.

\section{Multimedia Appendix 1}

CONSORT-EHEALTH checklist (V 1.6.1). 


\section{Multimedia Appendix 2}

Patient-Reported Outcome Measure Information System tools completed by the study subjects. [PDF File (Adobe PDF File), 97 KB-Multimedia Appendix 2]

\section{Multimedia Appendix 3}

Acceptance and commitment therapy-based automated mobile phone messaging protocol. [PDF File (Adobe PDF File), 44 KB-Multimedia Appendix 3]

\section{References}

1. Azar A. Renewal of Determination That A Public Health Emergency Exists. US Department of Health and Human Services. 2019. URL: https://www.phe.gov/emergency/news/healthactions/phe/Pages/covid19-21apr2020.aspx [accessed 2000-07-09]

2. Scholl L, Seth P, Kariisa M, Wilson N, Baldwin G. Drug and opioid-involved overdose deaths - United States, $2013-2017$. MMWR Morb Mortal Wkly Rep 2018 Jan 4;67(5152):1419-1427 [FREE Full text] [doi: 10.15585/mmwr.mm675152e1] [Medline: $\underline{30605448]}$

3. Brat GA, Agniel D, Beam A, Yorkgitis B, Bicket M, Homer M, et al. Postsurgical prescriptions for opioid naive patients and association with overdose and misuse: retrospective cohort study. Br Med J 2018 Jan 17;360:j5790 [FREE Full text] [doi: $\underline{10.1136 / \mathrm{bmj} . j 5790]}$ [Medline: 29343479]

4. Anthony CA, Volkmar A, Shah AS, Willey M, Karam M, Marsh JL. Communication with orthopedic trauma patients via an automated mobile phone messaging robot. Telemed J E Health 2018 Jul;24(7):504-509. [doi: 10.1089/tmj.2017.0188] [Medline: 29261036]

5. Scully RE, Schoenfeld AJ, Jiang W, Lipsitz S, Chaudhary MA, Learn PA, et al. Defining optimal length of opioid pain medication prescription after common surgical procedures. JAMA Surg 2018 Jan 1;153(1):37-43 [FREE Full text] [doi: 10.1001/jamasurg.2017.3132] [Medline: 28973092]

6. Deshpande PR, Rajan S, Sudeepthi BL, Nazir CP. Patient-reported outcomes: a new era in clinical research. Perspect Clin Res 2011 Oct;2(4):137-144 [FREE Full text] [doi: 10.4103/2229-3485.86879] [Medline: 22145124]

7. Ahmed S, Berzon RA, Revicki DA, Lenderking WR, Moinpour CM, Basch E, International Society for Quality of Life Research. The use of patient-reported outcomes (PRO) within comparative effectiveness research: implications for clinical practice and health care policy. Med Care 2012 Dec;50(12):1060-1070. [doi: 10.1097/MLR.0b013e318268aaff] [Medline: 22922434]

8. Review of Physician and Advanced Practitioner Recruiting Incentives. Merritt Hawkins. 2019. URL: https://www. merritthawkins.com/trends-and-insights/article/reports/

2019-review-of-physician-and-advanced-practitioner-recruiting-incentives/ [accessed 2020-07-09]

9. Ader DN. Developing the patient-reported outcomes measurement information system (PROMIS). Med Care 2007 May;45(Suppl 1):S1-S2 [FREE Full text] [doi: 10.1097/01.mlr.0000260537.45076.74]

10. Cella D, Choi SW, Condon DM, Schalet B, Hays RD, Rothrock NE, et al. PROMIS adult health profiles: efficient short-form measures of seven health domains. Value Health 2019 May;22(5):537-544 [FREE Full text] [doi: 10.1016/j.jval.2019.02.004] [Medline: $\underline{31104731]}$

11. Batten S. Essentials of Acceptance and Commitment Therapy. London, UK: Sage Publications; 2011.

12. Hayes S. Acceptance and commitment therapy, relational frame theory, and the third wave of behavioral and cognitive therapies - republished article. Behav Ther 2016 Nov;47(6):869-885. [doi: 10.1016/j.beth.2016.11.006] [Medline: 27993338]

13. Hughes LS, Clark J, Colclough JA, Dale E, McMillan D. Acceptance and commitment therapy (ACT) for chronic pain: a Systematic review and meta-analyses. Clin J Pain 2017 Jun;33(6):552-568. [doi: 10.1097/AJP.0000000000000425] [Medline: 27479642]

14. Dahl J, Wilson K, Nilsson A. Acceptance and commitment therapy and the treatment of persons at risk for long-term disability resulting from stress and pain symptoms: a preliminary randomized trial. Behav Ther 2004;35(4):785-801 [FREE Full text] [doi: 10.1016/S0005-7894(04)80020-0]

15. Powers MB, Vording MB, Emmelkamp PM. Acceptance and commitment therapy: a meta-analytic review. Psychother Psychosom 2009;78(2):73-80. [doi: 10.1159/000190790] [Medline: 19142046]

16. McCracken LM, Vowles KE. Acceptance and commitment therapy and mindfulness for chronic pain: model, process, and progress. Am Psychol 2014;69(2):178-187. [doi: 10.1037/a0035623] [Medline: 24547803]

17. Dindo L, Zimmerman MB, Hadlandsmyth K, StMarie B, Embree J, Marchman J, et al. Acceptance and commitment therapy for prevention of chronic postsurgical pain and opioid use in at-risk veterans: a pilot randomized controlled study. J Pain 2018 Oct;19(10):1211-1221 [FREE Full text] [doi: 10.1016/j.jpain.2018.04.016] [Medline: 29777950]

18. Abid Azam M, Weinrib AZ, Montbriand J, Burns LC, McMillan K, Clarke H, et al. Acceptance and commitment therapy to manage pain and opioid use after major surgery: preliminary outcomes from the Toronto general hospital transitional pain service. Can J Pain 2017 Jun 28;1(1):37-49. [doi: 10.1080/24740527.2017.1325317]

19. Anthony CA, Lawler EA, Ward CM, Lin IC, Shah AS. Use of an automated mobile phone messaging robot in postoperative patient monitoring. Telemed J E Health 2018 Jan;24(1):61-66. [doi: 10.1089/tmj.2017.0055] [Medline: 28622079] 
20. Anthony CA, Lawler EA, Glass NA, McDonald K, Shah AS. Delivery of patient-reported outcome instruments by automated mobile phone text messaging. Hand (N Y) 2017 Nov;12(6):614-621 [FREE Full text] [doi: 10.1177/1558944716672201] [Medline: 29091492]

21. Anthony CA, Peterson AR. Utilization of a text-messaging robot to assess intraday variation in concussion symptom severity scores. Clin J Sport Med 2015 Mar;25(2):149-152. [doi: 10.1097/JSM.0000000000000115] [Medline: 24905538]

22. Liu TC, Ohueri CW, Schryver EM, Bozic KJ, Koenig KM. Patient-identified barriers and facilitators to pre-visit patient-reported outcomes measures completion in patients with hip and knee pain. J Arthroplasty 2018 Mar;33(3):643-9.e1. [doi: 10.1016/j.arth.2017.10.022] [Medline: 29169689]

23. Scott EJ, Anthony CA, Rooney P, Lynch TS, Willey MC, Westermann RW. Mobile phone administration of hip-specific patient-reported outcome instruments correlates highly with in-office administration. J Am Acad Orthop Surg 2020 Jan 1;28(1):e41-e46. [doi: 10.5435/JAAOS-D-18-00708] [Medline: 31860543]

24. Anthony C, Polgreen L, Chounramany J, Foster E, Goerdt C, Miller M, et al. Outpatient blood pressure monitoring using bi-directional text messaging. J Am Soc Hypertens 2015 May;9(5):375-381 [FREE Full text] [doi: 10.1016/j.jash.2015.01.008] [Medline: 25771023]

25. Berrouiguet S, Baca-García E, Brandt S, Walter M, Courtet P. Fundamentals for future mobile-health (mhealth): a systematic review of mobile phone and web-based text messaging in mental health. J Med Internet Res 2016 Jun 10;18(6):e135 [FREE Full text] [doi: 10.2196/jmir.5066] [Medline: 27287668]

26. Hall AK, Cole-Lewis H, Bernhardt JM. Mobile text messaging for health: a systematic review of reviews. Annu Rev Public Health 2015 Mar 18;36:393-415 [FREE Full text] [doi: 10.1146/annurev-publhealth-031914-122855] [Medline: 25785892]

27. Day MA, Anthony CA, Bedard NA, Glass NA, Clark CR, Callaghan JJ, et al. Increasing perioperative communication with automated mobile phone messaging in total joint arthroplasty. J Arthroplasty 2018 Jan;33(1):19-24. [doi: 10.1016/j.arth.2017.08.046] [Medline: 29017803]

28. Jones KR, Lekhak N, Kaewluang N. Using mobile phones and short message service to deliver self-management interventions for chronic conditions: a meta-review. Worldviews Evid Based Nurs 2014 Apr;11(2):81-88. [doi: 10.1111/wvn.12030] [Medline: 24597522]

29. Eysenbach G, CONSORT-EHEALTH Group. CONSORT-EHEALTH: improving and standardizing evaluation reports of Web-based and mobile health interventions. J Med Internet Res 2011 Dec 31;13(4):e126 [FREE Full text] [doi: 10.2196/jmir.1923] [Medline: 22209829]

30. PROMIS® Scoring Manuals. Health Measures. URL: http://www.healthmeasures.net/index. php?option=com content\&view=article\&id=180\&Itemid=994) [accessed 2020-07-09]

31. Goz V, Anthony C, Pugely A, Lawrence B, Spina N, Brodke D, et al. Software-based postoperative communication with patients undergoing spine surgery. Global Spine J 2019 Feb;9(1):14-17 [FREE Full text] [doi: 10.1177/2192568217728047] [Medline: $\underline{30775203}$ ]

32. Tofte JN, Anthony CA, Polgreen PM, Buckwalter JA, Caldwell LS, Fowler TP, et al. Postoperative care via smartphone following carpal tunnel release. J Telemed Telecare 2020 May;26(4):223-231. [doi: 10.1177/1357633X18807606] [Medline: $\underline{30428766}$ ]

33. Nelson SE, Adams AJ, Buczek MJ, Anthony CA, Shah AS. Postoperative pain and opioid use in children with supracondylar humeral fractures: balancing analgesia and opioid stewardship. J Bone Joint Surg Am 2019 Jan 16;101(2):119-126. [doi: 10.2106/JBJS.18.00657] [Medline: 30653041]

34. Broderick JE, Schneider S, Junghaenel DU, Schwartz JE, Stone AA. Validity and reliability of patient-reported outcomes measurement information system instruments in osteoarthritis. Arthritis Care Res (Hoboken) 2013 Oct;65(10):1625-1633 [FREE Full text] [doi: 10.1002/acr.22025] [Medline: 23592494]

35. Bot AG, Bekkers S, Arnstein PM, Smith RM, Ring D. Opioid use after fracture surgery correlates with pain intensity and satisfaction with pain relief. Clin Orthop Relat Res 2014 Aug;472(8):2542-2549 [FREE Full text] [doi: 10.1007/s11999-014-3660-4] [Medline: 24777731]

36. Koehler RM, Okoroafor UC, Cannada LK. A systematic review of opioid use after extremity trauma in orthopedic surgery. Injury 2018 Jun;49(6):1003-1007. [doi: 10.1016/j.injury.2018.04.003] [Medline: 29704954]

\section{Abbreviations}

ACT: acceptance and commitment therapy

MME: morphine milliequivalents

POD: postoperative day

PRO: patient-reported outcome

PROMIS: Patient-Reported Outcome Measure Information System 
Edited by $G$ Eysenbach; submitted 09.01.20; peer-reviewed by L Dindo, $H$ Zhao; comments to author 05.03.20; revised version received 29.04.20; accepted 20.05.20; published 29.07.20

Please cite as:

Anthony CA, Rojas EO, Keffala V, Glass NA, Shah AS, Miller BJ, Hogue M, Willey MC, Karam M, Marsh JL

Acceptance and Commitment Therapy Delivered via a Mobile Phone Messaging Robot to Decrease Postoperative Opioid Use in

Patients With Orthopedic Trauma: Randomized Controlled Trial

J Med Internet Res 2020;22(7):e17750

URL: https://www.jmir.org/2020/7/e17750

doi: $\underline{10.2196 / 17750}$

PMID: $\underline{32723723}$

(C) Chris A Anthony, Edward Octavio Rojas, Valerie Keffala, Natalie Ann Glass, Apurva S Shah, Benjamin J Miller, Matthew Hogue, Michael C Willey, Matthew Karam, John Lawrence Marsh. Originally published in the Journal of Medical Internet Research (http://www.jmir.org), 29.07.2020. This is an open-access article distributed under the terms of the Creative Commons Attribution License (https://creativecommons.org/licenses/by/4.0/), which permits unrestricted use, distribution, and reproduction in any medium, provided the original work, first published in the Journal of Medical Internet Research, is properly cited. The complete bibliographic information, a link to the original publication on http://www.jmir.org/, as well as this copyright and license information must be included. 